writings, all of which can be seen in the Library. Information on specific aspects of College history can be obtained by consulting the Library's archival database.

\section{Histories of the 'College'}

ANON (n.d. but about 1960) a historical note regarding the Royal Medico-Psychological Association. 4 pages.

Rollin, H. R. (1987) The Royal College of Psychiatrists, London, RCP.

TUKE, D. HACK (1881) 1841-1881 (presidential address). Journal of Mental Science, 27, 305-342.

WALK, A. (1978) "Forty years of wanderings" - the Medico Psychological Association, 1855-1894. British Journal of Psychiatry, 132, 530-547.

- \& WALKER, D. L. (1961) Gloucester and the beginnings of the RMPA. British Journal of Psychiatry, 107, 603-631.

- (1973) The Royal College of Psychiatrists. St Batholomew's Hospital Journal, 77, 135-139.

- (n.d.) The Royal Medico-Psychological Association: a historical note. 4 pages.

WooD, T. O. (1896) The early history of the MedicoPsychological Association. Journal of Mental Science, 52, 241-260.

\section{Donations}

Members have continued to be most generous in giving copies of their published works to the Library.

During the last six months books have been gratefully received from the following members:

Drs A. W. Bateman and D. Sturgeon UCH Textbook of Psychiatry: an integrated approach

Dr G. Beaumont Benzodiazepines: current concepts: biological, clinical and social perspectives

Professor P. J. V. Beumont Textbook of Psychiatry

Dr J. M. Bird The Burden: fifty years of clinical and experimental neuroscience at the Burden Neurological Institute

Professor R. Bluglass and Dr P. Bowden Principles and Practice of Forensic Psychiatry

Dr S. Bourne Under the Doctor: studies in the psychological problems of Physiotherapists

Dr J. Bowlby Charles Darwin: a new biography

Professor G. W. Brown Life Events and Illness

Dr T. M. Brown Handbook of Emergency Psychiatry

Dr J. Ellard Some Rules for Killing People: essays on madness, murder and the mind

Dr D. Enoch Schizophrenia: voices in the dark: hope for those who care

Dr K. W. M. Fulford Moral Theory and Medical Practice

Dr I. M. Goodyer Life Experiences, Development and Childhood Psychopathology

Dr J. D. Haldane Wedlocked?
Dr L. K. G. Hsu Eating Disorders: Recent Developments in Adolescent Psychiatry

Dr J. Jancar Annual Report on Publications, Research and Other Events to Frenchay HA, SWRHA and University of Bristol

Dr R. Littlewood Aliens and Alienists: ethnic minorities and psychiatry, 2nd ed.

Dr M. R. Oates Psychological Aspects of Obstetrics and Gynaecology

Drs E. H. Reynolds and M. Trimble The Bridge between Neurology and Psychiatry

Dr H. Rollin Festina Lente: a psychiatric odyssey

Dr M. J. Sainsbury Sainsbury's Key to Psychiatry, 4thed.

Dr N. Sartorius Health and Behaviour: selected perspectives

Dr A. C. R. Skynner Explorations with Families; Institutes and How to Survive Them

Dr D. A. Spencer Record of the Meanwood Park Hospital Archives

Dr D. Stafford-Clark Psychiatry for Students, 7th ed.

Dr A. Stevens On Jung; Archetype: a natural history of the self

Dr D. Waxman Hypnosis: 4th European congress at Oxford

Dr M. Weller International Perspectives in Schizophrenia: biological, social and epidemiological findings

SUSAN FLOATE

Librarian

\section{Examination results}

\section{MRCPSYCH PART I - April 1990}

Out of 309 candidates who sat the examination, 166 passed.

Paul Douglas Addison; Oyebode Adegboyega Adesida; Shakeel Ahmad; Waheed Mohamed Leon L K Ahmed; Seniya Akhtar; Abdel Moniem Abdelbaki Ali; Elizabeth Anne Almond; Frances Elizabeth Margaret Anderson; Pernia Arshad; Petrus Jacobus Cornelius Bakx; Joanne Barton; Natasha Bijlani; Helen Mary Birchall; Jonathan Ian Bisson; Ann Karen Blagden; Catherine Sarah Bowen; Jocelyn Alan Bray; John Stedman Brewin; Maria Broughton; Philip Morrison Brown; Phillip Michael Brown; Roger Alan Bullock; Jeremy Arthur Burley; Alyson Fleur Callan; Chan Sai Yin; Ben Cheung Kin Leung; Barry Benjamin Chipchase; Tariq Mahmood Choudhry; Dicky Chung Wai Sau; Joan Elizabeth Clark; Maria Del Carmen Clemente Meoro; Mark Thomas Cohen; Maria Elena Colomina Elias; Louise Mary Geraldine Connolly; Alison Emma Conway; Christopher Daniel Corcos; Peter Leonard Cornwall; 
Stuart Jeremy Cox; Vanessa Louise Crawford; Caitriona Alicia Shiobhaun Crowe; Sylvia Dahabra; Rina Datta Gupta; Owen Meredith Davies; Richard Anthony Davies; Rachel Jane Nesbitt Davis; Sheila Catherine Dawson; Catherine Eluned Dorkins; Athanassois Douzenis; Mateen Durrani; Nigel John Bromely Eastwood; David Elwell; Alexander Esterhuyzen; Rafael Euba Ibarreche; Richard Morgan Evans; Victoria Farrer; Christopher Frank Fear; Gearoid Fitzgerald; John Franklin; Moya Catriona Gear; Janice Louise Gillow; Jonathan Mark Godfrey; Rainer Goldbeck; Valerie Anne Goodier; Sheila Muriel Janet Gordon; Anne Denise Hall; Gillian Harrison; Isobel Heyman; Jonathan Charles Hillam; Claire Fiona Hilton; David John Holmes; Lynn Ann Hutchinson; Hwang Kim Shyan; Gabriel Obukohwo Ivbijaro; Teerakiat Jareonsettasin; Dinah Jayson; Alison Jenaway; James Johnston; Alison Margaret Jones; Heledd Mair Jones; Mark Bryan Jones.

Ciaran Peter Kelly; Francis John Kelly; Joanne Louise Kennedy; King Han Kho; Andrew Philip Joseph Kinch; Keren Anne King; John Andrew Knapp; Palnati Krishna Reddy; Leopold Kroll; Andrew William Lawrie; Kenneth Douglas Lindsay; Philippa Anne Loan; Philip Anthony Lucas; Aslog Kristina Malmberg; Laura Mary Catherine Mannion; Cecilia Mary Marshall; Pedro Mascunan Perez; Simon Thomas Masterson; Inas Youssef Aziz Matta; Katrina Mary Mayo; Kathleen Rosemary McIntyre; Nirmalie Varini Benigna Mirando; Maria Leonor Montoliu Tamarit; Maria Katharina Irmgard Mueller; Janet Fiona Muir; Suniti Mukerji; Timothy Giff Nicholson; Andrew Nicol; John Christopher Ohlsen; Neil Trevor Poyntz Owen; Karen Susan Palmer; Stewart Bertrand Geoffrey Park; David Charles Parker; Sara Frances Parslow; Bienvenido Presilla Linero; Mark Pummell; Nimishchandra Natverlal Purohit.

Digby John Quested; Louise Anne Quinn; Manoranjitham Raveendran; Ijaz-Ur Rehman; David Reiss; Josephine Anne Richards; Megan Charlotte Roberts; Carol Robertson; Shauna Deirdre Elaine Rudge; Roberto Rusca; Amany Anton Gergs Saad; Tariq Yusuf Saraf; Linda Elizabeth Schofield; Hilary Jane Scurlock; Luis Miguel Segovia Lopez; Manohary Senathirajah; Shirley Shakespear; Stephen William Simpson; Karmen Slaveska; Cameron Lawson Smith; Janet Marie Smith; Christina Sonneborn; Ghulam Mustafa Soomro; Frederick Philip Souza Faria; Christopher John Speller; Quentin Wynn Spender; Ekkenhart Friedrich Staufenberg; Jill Nicola Stemman; Alison Wendy Stevenson; Suzanne Marie Stewart; Catriona Mhairi Ann Stickle; Marios Strouthos; Julia Catherine Stubbs; Ariamalar Suntharanathan; Pravin Asrajit Thevathasan; Blanca Ugarte Alvarado; Hendrik Van Den Brink; Morris David
Vinestock; Willem Visser; Hilary Anne Vowles; Steven William Welsh; Roger David Wesby; Jane Fiona Whittaker; Julie Catherine Wild; Guy Andrew Windsor; Michael Wong Ming Cheuk; Clare Jane Wood; Neil Richard Wright; Peter Andrew Wynn Owen.

\section{Membership examination - May 1990}

Out of 46 candidates who sat the examination, 28 passed.

Ponnambalam Balakrishnan; Sylvia Rochelle Bloomberg; Gerard Michael Burke; Kevin Robert Calder-Potts; Kurudi Chandrashekar; Jean Catherine Damms; Richard Philip Evans; David Fainman; Paul Michael Myrie Foster; Therese Joan Gunning; Moyra Frances Guthrie; Sigrid Herold; Susan Elisabeth Jenkins; Christine Mary MacGregor; Gillian Suzanne McLean; Janet Lesley Mead; Eamonn Francis Moloney; Elizabeth Jane Brenda Morris; Michael John Mullan; Carol Susan Page; Michael David Radford; Jean Perry Smillie Reid; Stephen Kim Rossiter; John Joseph Scanlon; Vincent Anthony Sharkey; Jean Maria Sherrington; David Henry Stainthorp; Deirdre Frances Stone.

\section{MRCPSYCH PART II - May 1990}

Out of 245 candidates who sat the examination, 121 passed.

Stephen John Aldridge; James Barclay Anderson; Belito Jex Arana; Martin Roger Baggaley; John Stephen Barnes; Roderick John Beasley; Peter Andrew James Bell; Christopher John Bench; Esther Rose Lynn Bennett; Colin Anthony Biggins; William Black Christine Joy Boardman; Anne Margaret Boocock; Joanna Talbot Bowen; Joseph Sim Bowie; Oonagh Noreen Bradley; Margaret Hamilton Bremner; Archibald Stephen Brown; Derek Robin Pritchard Brown; William Dennis Alexander BruceJones; Timothy Bullock; Chee Li Chang; Eric Chen Yu Hai; Nicholas Alexander Howard Clarke; Robert Maurice Cohen; Alison Susan Conway; Colm Cooney; Edan Patrick Corcoran; Paul Crichton; Elizabeth Mary Joseph Cryan; Elizabeth Jane Cusworth; Timothy John Dalkin; Alexandra Davis; Prasanna Nemichandra De Silva; Margaret Catherine Dolan; Anita Duggal; Yvonne Grace Edmonstone; Mary Marjorie Ellis; Mary Elizabeth Ferguson; Claire Barbara Flannigan; Sheila Russell Flett; Thomas John Foster.

Benjamin Hugh Green; Nancy Mary Greenhalgh; Richard Huw Griffiths; Peter David Halford; David John Hall; Shuja Haque; Deborah Angela Harrison; 
Judith Anne Harrison; Bernard Jonathan Hill; Janis Hillier; Barbara Lindsay Houghton; Anne Lesley Jasper; Sudad Sadiq Mohamed Jawad; Gwyneth Eleanor Siobhan Jeffreys; Peter Brian Jones; Rajinder Judge; Olatunji Junaid; Anastassios Kalofonos; Robert Francis Kehoe; Margaret Kelleher; Alison Mary Kelly; Tracey Lees; Sudesh Kumar Lekh; Philip David Rhys Lewis; James Vincent Lucey; Caroline Janet Luke; Christopher Maloney; Ivana Markova; Vallakalil Mathew Mathew; Keith Matthews; Sara Helen Margaret Matthews; Joseph William McDonald; Fiona Christine Patricia NcNicholas; Gavin William Mercer; Peter Brian Moore; Kathryn Amy Langton Morgan; Leigh Anthony Neal; Patrick Gerald O'Mahony; Elaine Catherine Ogg; Derek Edward Palmer; Matthew Paul Hugh Patrick; Marguerite Marie A A Peutz; Nigel Luke Prior.

Julian Hilton Race; Robert Greig Ramsay; Margaret Ellen Haslam Rangecroft; Ian Robert Rayner; Graham Thomas Ridley; Angela Judith
Roberts; Jeremy Mark Roberts; Stewart John Roberts; Christopher Edward Robson; Graham Donald Sanderson; Robert Clive Schapira; Hugh George Series; William David Shapland; Clifford William Sharp; Catherine Susan Shaw; Kamil Mohamed Sidahmed; Rebecca Jane Smale-Adams; Marcellino Gerard Mooney Smyth; Ann Elizabeth Snedden; Shankarnarayan Srinath; Paul St John Smith; Cameron Ross Stark; Anita Rita Timans; Brian Robert Timney; Helen Margaret Kirby Towers; Katherine Louise Towlson; David Tsang Fan Kwong; Stewart David Vaggers; Alisa Wacharasindhu; David Gerard Walshe; Stephen John Warren; Elizabeth Mary Whitfield; David Vaughan Williams; Michael Wong Tak Hing; Tracey Oriole Woods; Michael Yiu Gar Chung.

\section{Prizewinner}

The Laughlin Prize has been awarded to Dr E. J. B. Morris in the Membership Examination.

\section{Obituary}

Editor: Henry R. Rollin

JOHN RICHARD HAMILTON, formerly Medical Director, Broadmoor Hospital and Honorary Senior Lecturer in the Department of Forensic Psychiatry, Institute of Psychiatry, London SES

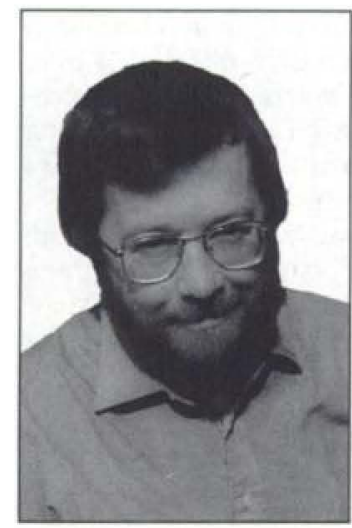

John Hamilton was born in Berkshire in October 1943, just a few miles away from where he died. In the interim he achieved much. He had the privilege of a Scottish education through school and university. He completed important research, followed a distinguished clinical career and contributed substantially to the Royal College of Psychiatrists. He married Mary Connor, whose devotion ensured the strong start to his career and its consolidation. She survives him with their two fine sons. This year he formalised his second marriage to Henrietta Bullard, who was his greatest support over the last few years. In the last nine months of his life, John was aware of his likely death sentence but nevertheless fought, not only to live, but also to continue a significant contribution to the subject he loved. He was leading the launch of research into the real security needs of patients in the special (maximum security) hospitals. The dignity and stoicism with which he met his illness and its intrusive treatment, and the extent to which he succeeded in maintaining his creativity in work was so successful that he forced most of us to forget the gravity of his condition much of the time. In his last week he had arranged a meeting with his closest research colleagues, sadly that meeting never took place: he died on 2 June 1990.

The importance of his work outlives him. It lives in his students, from a wide range of disciplines, to whom he was a wise teacher. It lives in his wider academic contribution, writing and reviewing for 